\title{
Gel Phase Vesicles Buckle into Specific Shapes
}

\author{
François Quemeneur, ${ }^{1}$ Catherine Quilliet, ${ }^{2, *}$ Magalie Faivre, ${ }^{3}$ Annie Viallat, ${ }^{4}$ and Brigitte Pépin-Donat ${ }^{1, \dagger}$ \\ ${ }^{1}$ UMR5819 SPrAM (CEA-CNRS-Univ. Grenoble)/INAC/CEA-Grenoble, France \\ ${ }^{2}$ Univ. Grenoble/CNRS, LIPhy UMR5588, Grenoble, France \\ ${ }^{3}$ Institut des Nanotechnologies de Lyon, UMR5270 CNRS/Univ. Lyon 1, France \\ ${ }^{4}$ Laboratoire Adhésion et Inflammation, CNRS UMR7333, Inserm UMR1067, Aix-Marseille Université, France
}

(Received 23 November 2010; published 9 March 2012)

\begin{abstract}
Osmotic deflation of giant vesicles in the rippled gel phase $P_{\beta^{\prime}}$ gives rise to a large variety of novel faceted shapes. These shapes are also found from a numerical approach by using an elastic surface model. A shape diagram is proposed based on the model that accounts for the vesicle size and ratios of three mechanical constants: in-plane shear elasticity and compressibility (usually neglected) and out-of-plane bending of the membrane. The comparison between experimental and simulated vesicle morphologies reveals that they are governed by a typical elasticity length, of the order of $1 \mu \mathrm{m}$, and must be described with a large Poisson's ratio.
\end{abstract}

DOI: 10.1103/PhysRevLett.108.108303

PACS numbers: $82.70 . \mathrm{Uv}, 62.20 .-\mathrm{x}$

Probing the structural and mechanical properties of soft shells by noncontact techniques is a challenging approach in soft matter and in cell biology, where contacts may trigger surface and/or cell adhesion and bias results [1]. For instance, morphological changes of fluid-phase lipid vesicles under osmotic or temperature variations have been largely studied for the past 30 years. They have shown that vesicle shapes are governed by the bending energy, the spontaneous curvature of the two monolayers of the membrane [2] and by their area difference [3]. Surprisingly, very few studies have concerned the shapes of gel-phase vesicles [4-6]. In addition to the bending stiffness and the stretching elasticity, the existence in the gel state of a lipid bilayer of a nonzero shear modulus is likely to generate specific deformations and new vesicle shapes. This was indeed observed in the model of coupled bilayer cytoskeleton proposed in [7-9] for red blood cells, and in the buckling instability that occurs under large local external forces on actin-coated [10] and on gel-phase vesicles [11]. Here, we report observations of buckling induced by a nonlocal constraint on gel-phase giant unilamellar vesicles (GUVs, diameter $>500 \mathrm{~nm}$ ) upon deflation induced by applying an isotropic osmotic pressure. We propose a simple model that captures the major observed morphologies. The study highlights the relationship between the elastic properties of the lipid membrane and the specific faceted shapes taken by the vesicles.

Deflation experiments were performed on DMPC $(1,2-$ dimyristoyl-sn-glycero-3-phosphocholine) GUVs in the rippled gel phase $P_{\beta^{\prime}}$ at $15^{\circ} \mathrm{C}$. GUVs were prepared by electroformation [12] above the main acyl chain crystallization temperature $T_{m}=23.6^{\circ} \mathrm{C}$ [13] in a $100 \mathrm{mM}$ sucrose solution, and by slowly decreasing the temperature down to $15^{\circ} \mathrm{C}$ with a cooling rate of $0.05{ }^{\circ} \mathrm{C} / \mathrm{min}$. In order to prevent the breaking of the lipid membrane at the transition, the volume of vesicles was decreased to adjust to their loss of surface area $(\sim 28 \%$ between the $L_{\alpha}$ fluid and the $P_{\beta^{\prime}}$ rippled phases [14]) by adding a controlled sucrose solution in the external solution. Gelphase GUVs obtained with this protocol were spherical and presented no observable defects in the membrane. Finally, GUVs sedimented in an iso-osmolar glucose solution were kept at $15^{\circ} \mathrm{C}$ and osmotically deflated by adding controlled amounts of glucose solution of suitable concentration in the external solution. GUVs were observed by phase contrast microscopy. The obtained shapes displayed in Fig. 1 line (a) show obvious differences with the classical shapes observed on vesicles in the fluid state [15]. Subjected to the osmotic shock, gel-phase GUVs shrink and develop a large variety of morphologies, from stomatocytes to concave polyhedra (i.e., sphere paved with depressions). The final faceted state is reached around $40 \mathrm{~min}$ after the beginning of the deflation (the whole process is limited by diffusion of glucose molecules in the surrounding medium), and, thereafter, no shape modification is observed over several hours, when temperature and osmolarity are kept constant.

In order to quantitatively understand these specific shapes, we model the 2D gel-phase membrane by a surface with an in-plane Hooke elasticity [16] determined by two 2D phenomenological constants, the Young modulus $Y_{2 \mathrm{D}}$ and the 2D Poisson's ratio $\nu_{2 \mathrm{D}}$, and by an out-of-plane bending elasticity. We describe the bending contribution by the Helfrich model [2] that involves only two constants, the spontaneous curvature $C_{0}$ and the bending modulus $\kappa$ of the membrane. An initial vesicle is considered as a spherical surface of radius $R$, enclosing a volume $V_{0}$. As the vesicle remains spherical during the phase transition towards the $P_{\beta^{\prime}}$ phase, we consider that the vesicle remains unstrained, which implies $C_{0}=2 / R$. Dimensional analysis reveals that three dimensionless parameters control the shape of the vesicle when its volume decreases from $V_{0}$ to 


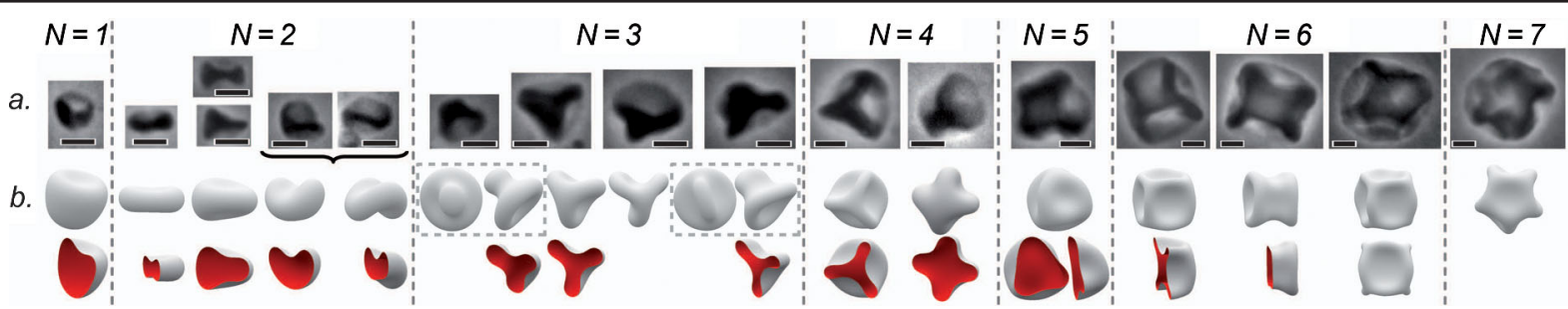

FIG. 1 (color online). (a) Experimental shapes for deflated gel-phase GUVs $\left(v_{r}=0.6\right)$ for increasing radii. Black scale bar: $5 \mu$ m. (b) Numerical simulations: each shape is characterized by the number of depressions $N$ (see text). $N=0$ : sphere, oblate, untwined chestnut; $N=1$ : stomatocyte; $N=2$ : discocyte, asymmetric discocyte, bean, crisp; $N=3$ : nipple, 3 blades (or knizocyte), twisted 3 blades, bladed nipple; $N=4$ : tetrahedron, 4 blades. $N=5$ : dumbbell with triangular leg; $N=6$ : cube, dumbbell with square leg, bulged cube; $N=7$ : dumbbell with 5 -star leg.

$V$ : the deflation $\frac{\Delta V}{V}=\frac{V_{0}-V}{V_{0}}=1-v_{r}\left(v_{r}\right.$ is the reduced volume), the Föppl-von-Kármán number $\gamma=\frac{Y_{2 \mathrm{D}} R^{2}}{\kappa}$ $[17,18]$, and the Poisson's ratio $\nu_{2 \mathrm{D}}$ (maximum value 1 , for incompressible surfaces). The numerical study is performed by reducing the volume of the initial vesicle in small steps $\left(\approx 0.6 \%\right.$ of $\left.V_{0}\right)$, searching at each stage an equilibrium shape with the SURFACE EVOLVER software as presented in [19]. This quasistatic deflation has been simulated for a wide range of parameters $\left(0 \leq \frac{\Delta V}{V} \leq 0.7 ; 1.8 \leq\right.$ $\gamma \leq 2430 ; 0 \leq \nu_{2 \mathrm{D}} \leq 0.98$ ). Values of $\gamma$ well below $10^{4}$ ensure the absence of singularities due to the intrinsic defects of the numerical mesh [20]. Two typical sequences of deflation are shown in Fig. 4(b), paths 1 and 2. The spherical symmetry of the vesicle is first conserved under small deflation. Then concave facets (or depressions) appear on the vesicle. The facets proliferate (number of facets $N_{\text {transient }}$ ) with a further volume reduction, until they completely pave the surface of the vesicle. The shapes are then characterized by a maximum number $(N)$ of facets. A subsequent deflation only affects the concavity of the facets. These faceted shapes, consistent with experimental observations, are associated with local minimum energy values [19]. Energy considerations are detailed in Supplemental Material [21]: stretching and bending energies of faceted shapes increase with deflation. The total energy of metastable multifaceted shapes is higher than that of bowl shapes (single depression); tendency also observed in Ref. [18] where $\nu=\frac{1}{3}$. Typically, there is a factor 1 to 5 when the number of facets increases from 1 to 6 . We then explored the metastability lines related to multifaceted conformations in the $\left(\frac{\Delta V}{V}, \gamma, \nu_{2 \mathrm{D}}\right)$ space. Vesicles sufficiently deflated to have the maximum number of facets succeed each other always in the same order upon increasing their radius, as illustrated in Fig. 1 line (b). This succession provides a way to quantify the shapes: for some of them indeed (discocyte, 3-blades, tetrahedron, cube, etc.), it is possible to unambiguously determine $N$. When the notion of number of facets becomes questionable (e.g. bean, nipple), an indirect attribution can be done by continuity in the succession. For $N>6$, shapes are concave polyhedra, bulged (i.e., with a protuberance on the rims that separate two faces) or not. For $N=6,8,12,20$, vesicle shapes display soft regular polyhedra as in the case of viruses [17] and desiccated pollens [22].

This quantitative shape description allows us to study numerically the influence of $\gamma$ and $\nu_{2 \mathrm{D}}$ on $N$. As shown in Fig. 2, for all $\nu_{2 \mathrm{D}}$ ranging between 0 and $0.98, N$ gathers on a quasilinear master curve as a function of $\sqrt{\gamma / 12\left(1-\nu_{2 D}^{2}\right)}$. Dimensionally, this latter quantity can be considered as a reduced radius $R / d_{\text {eq }}$, where $d_{\mathrm{eq}}=\sqrt{12\left(1-\nu_{2 \mathrm{D}}^{2}\right) \kappa / Y_{2 \mathrm{D}}}$ is homogeneous to a length. Within the frame of thin shells deformation theory, this scaling law can easily be understood [16]. A thin isotropic shell of thickness $d$ and radius $R$ submitted to a uniform pressure buckles by reversion of a spherical cap of size $L \approx \sqrt{d R}$ [16]. The maximum number of facets that pave the full surface of the initial sphere therefore scales like $N \propto \frac{R^{2}}{L^{2}} \approx \frac{R}{d}$. This relation replaced in a 3D context yields the numerical scaling obtained in Fig. 2. It is important to

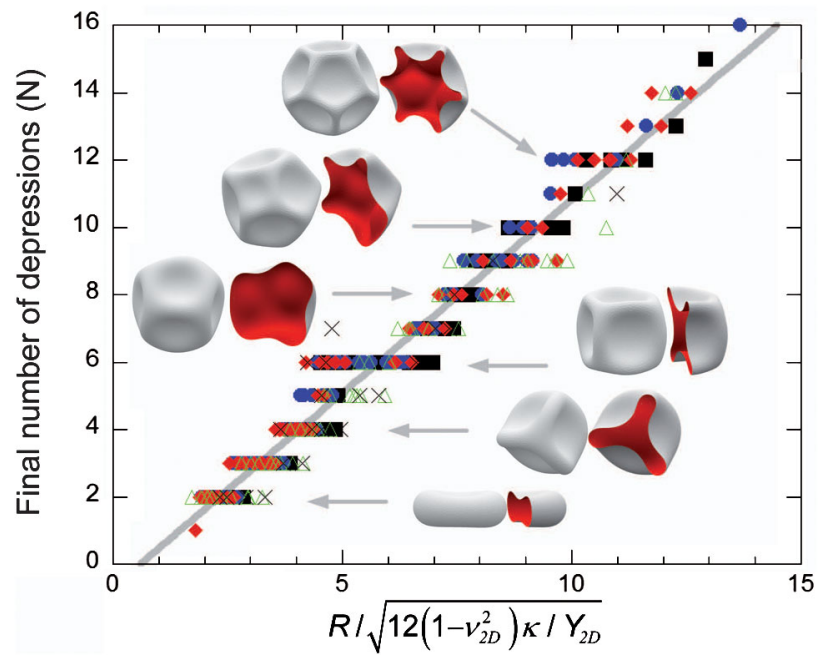

FIG. 2 (color online). SURFACE EVOLVER simulations: variation of $N$ with the reduced radius $R / d_{\text {eq }}$. Black $\square: \nu_{2 \mathrm{D}}=0$ to 0.25 ; blue 0 : $\nu_{2 \mathrm{D}}=0.3$ to 0.5 . red $\diamond: \nu_{2 \mathrm{D}}=0.55$ to 0.75 . Green $\triangle$ : $\nu_{2 \mathrm{D}}=0.8$ to 0.90 . Gray $\times: \nu_{2 \mathrm{D}}=0.92$ to 0.98 . Master curve: for $R>0.59 d_{\text {eq }}, N=1.15\left(R / d_{\mathrm{eq}}-0.59\right)$ (gray line). 
note that (i) this scaling law keeps its validity for a range of parameters much larger than those valid for a thin shell of an isotropic material (case which reduces to $\nu_{2 \mathrm{D}}=\nu_{3 \mathrm{D}}<\frac{1}{2}$ [19] and $\gamma \gg 1$ in the linear approximation), and (ii) the nonzero shear energy of the membrane is responsible for the existence of a typical length of deformation, while in systems only governed by the bending energy, the only length scale is the radius of the object [15]. For $\nu_{2 \mathrm{D}}>\frac{1}{2}$ (maximum value for bulk materials), $d_{\mathrm{eq}}$ has no direct 3D equivalent. It is not necessarily a thickness, but a characteristic elastic length of the membrane, that gives the typical size of the deformations on the sphere: $\sqrt{d_{\mathrm{eq}} R}$.

Making up for the lack of experimental 3D images, experimental values of $N$ were determined by comparing phase contrast microscopy observations to numerical shapes. Figure 3 shows a plot of $N$ measured in this way as a function of the initial GUV radius for three reduced volumes and more than 1300 vesicles. In all cases, the number of facets on the vesicles had reached its maximum value and remained constant upon further deflation. The variation of $N$ with $R$ is consistent with the numerical linear dependence obtained previously in Fig. 2, and allows the experimental determination of $d_{\mathrm{eq}} \sim 1.8 \mu \mathrm{m}$. This value is several orders of magnitude greater than both bilayer thickness $(\sim 5 \mathrm{~nm})$, and periodic undulations of the rippled phase (amplitude $\sim 1-11 \mathrm{~nm}$ and wavelength $\sim 15-55 \mathrm{~nm}$ ) [23]. Therefore, despite their relatively small thickness, the vesicles in gel phase cannot be regarded as "thin shells" (i.e., "of an isotropic material"), where $d_{\text {eq }}$ is

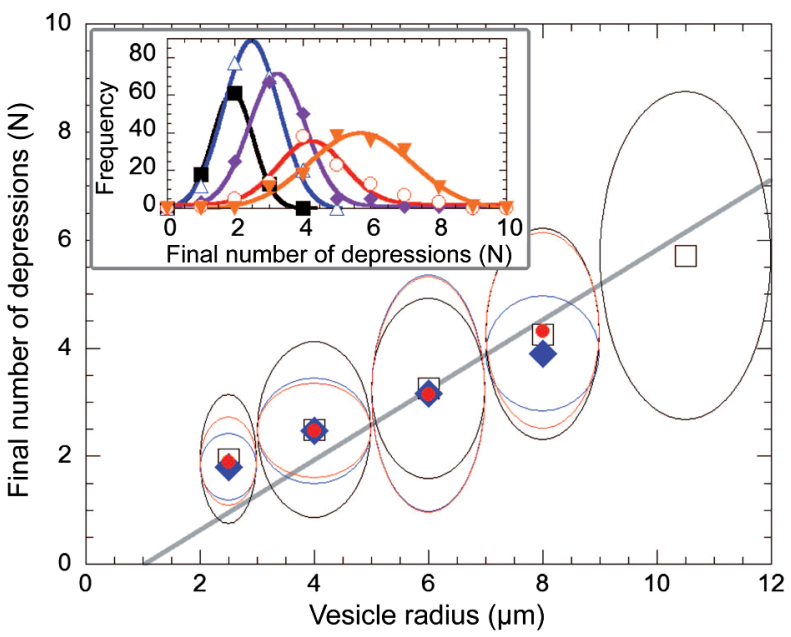

FIG. 3 (color online). Experimental value of $N$ as a function of the GUV radius. The inset shows the occurrence of $N$ for several ranges of radii (black, $\square$ : $2-3 \mu \mathrm{m}$; green, $\triangle$ : 3-5 $\mu \mathrm{m}$; purple, ४: 5-7 $\mu \mathrm{m}$; red, $\bigcirc: 7-9 \mu \mathrm{m}$; orange, $\nabla: 9-12 \mu \mathrm{m})$, for $v_{r}=$ 0.6; solid lines are Gaussian fits. r.m.s. values of each size distribution are taken as the vertical error bar in the main diagram. Black $\square$ : $v_{r}=0.6$; blue $\diamond: v_{r}=0.45$; red $\bullet$ : $v_{r}=$ 0.35. Curves drawn for different $v_{r}$ show no notable differences. The gray line is the master curve of Fig. 2 with $d_{\mathrm{eq}} \sim 1.8 \mu \mathrm{m}$. the thickness. This typical elastic length can be rewritten $d_{\mathrm{eq}}=\sqrt{6\left(1+\nu_{2 \mathrm{D}}\right) \kappa / \chi_{2 \mathrm{D}}}$, where $\chi_{2 \mathrm{D}}$ is the elastic area compressibility (or "stretching") modulus. By taking $\kappa \sim 100 k_{B} T$ [14], we find $\chi_{2 \mathrm{D}} \approx 1 \mu \mathrm{N} / \mathrm{m}$. This value is very weak compared to that given in [14], which corresponds to partial unfolding of the ripples and was measured by micropipette aspiration on vesicles weakly tensed, where undulations at a scale larger than ripples were flattened out. Our low value of $\chi_{2 \mathrm{D}}$ might be linked to fluctuations at a mesoscopic scale, larger than the individual ripples size but smaller than the vesicle radius. In the absence of a specific theory for the fluctuations of solid membranes, our study, which unambiguously shows a micron-size value for the characteristic length of deformation, provides a clue for a possible entropic origin of the area compressibility modulus.

The diagram of vesicles morphology, determined numerically and characterized by the number of facets, either $N_{\text {transient }}$ or $N$, is represented in Fig. 4 in the plane $\left(v_{r}\right.$, $R / d_{\mathrm{eq}}$ ) for three values of $\nu_{2 \mathrm{D}}$. It displays two clearly distinct zones: the $N$-domain where the number of facets has reached its maximum (in which one should find the experimental morphologies of Fig. 1), and the $N_{\text {transient }}$ domain. The coincidence of both experimental and numerical $N$ domains requires that $\nu_{2 \mathrm{D}}$ is at least equal to 0.8. Its maximum acceptable limit is 0.95 , for which shapes differ from those displayed in Fig. 1 (e.g., depressions are surrounded by spicules; these poorly compressible surfaces will be treated in a subsequent publication).

This high value of Poisson's ratio value confirms the fact that gel-phase GUVs cannot simply be regarded as thin shells of isotropic bulk material [16], where $\nu_{2 \mathrm{D}}=\nu_{3 D} \leq$ 0.5 . The discrepancy between the lipid membrane thickness and the typical elasticity length may be understood by the anisotropic nature of the constitutive material, i.e., the rippled lipid bilayer, that has different properties in its average plane, and in the perpendicular direction. The agreement between experimental and numerical vesicle shapes nevertheless shows the relevancy of this $2 \mathrm{D}$ elastic model based on in-plane isotropy, shear modulus and Helfrich curvature energy [24]. Our simulations show a universal sequence of shapes and provide an alphabet to quantitatively interpret deflated morphologies in various experimental systems. More generally, the simulations reveal that the Poisson's ratio, which generally varies over a narrow range of values and is then often neglected in favor of $\gamma$ in thin shell descriptions, has a crucial role when it approaches 1 . Our study explores a wide range of elastic constants suitable to describe many materials, from thin shells of isotropic material $\left(\nu_{2 \mathrm{D}} \leq 0.5\right)$ to surfaces with no shear elasticity $\left(\nu_{2 \mathrm{D}} \approx 1\right)$, like fluid vesicles. Moderate values of the Föppl-von Kármán constant and small spontaneous curvatures are in a range of values different from that involved in transitions of viral shells, and act in another way on the object shapes [20]. Besides 


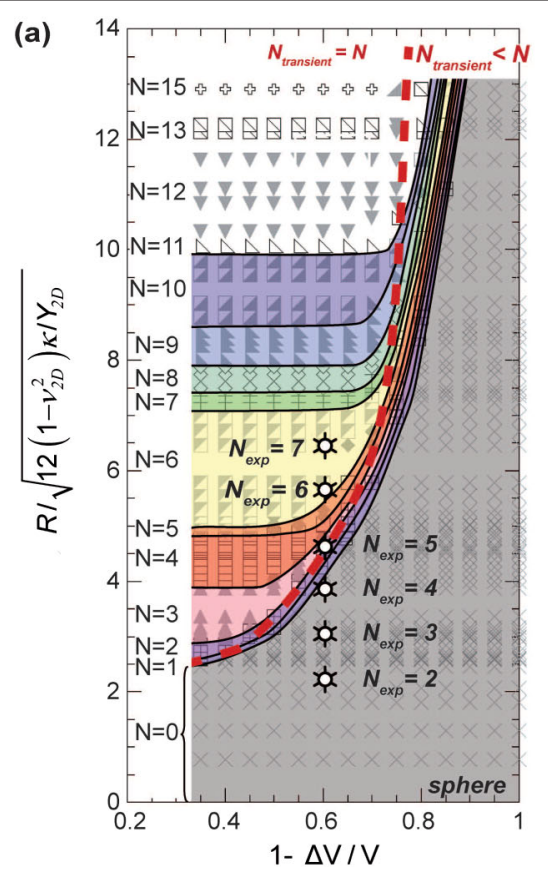

(b)

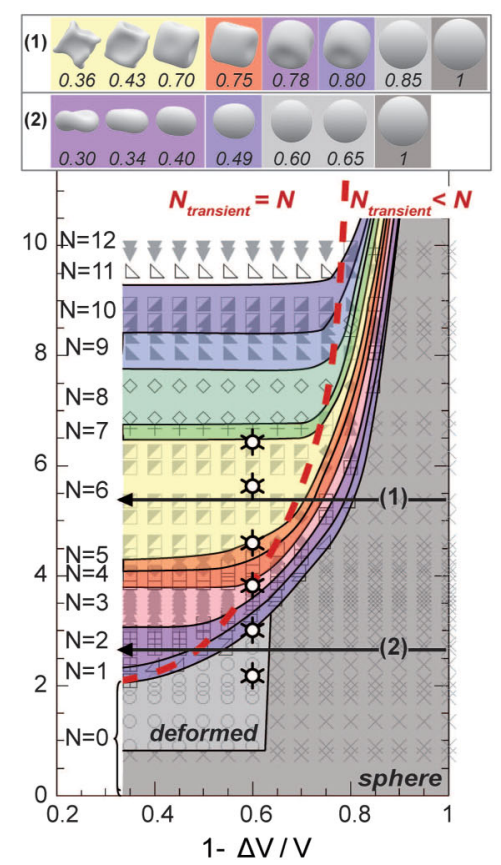

(c)

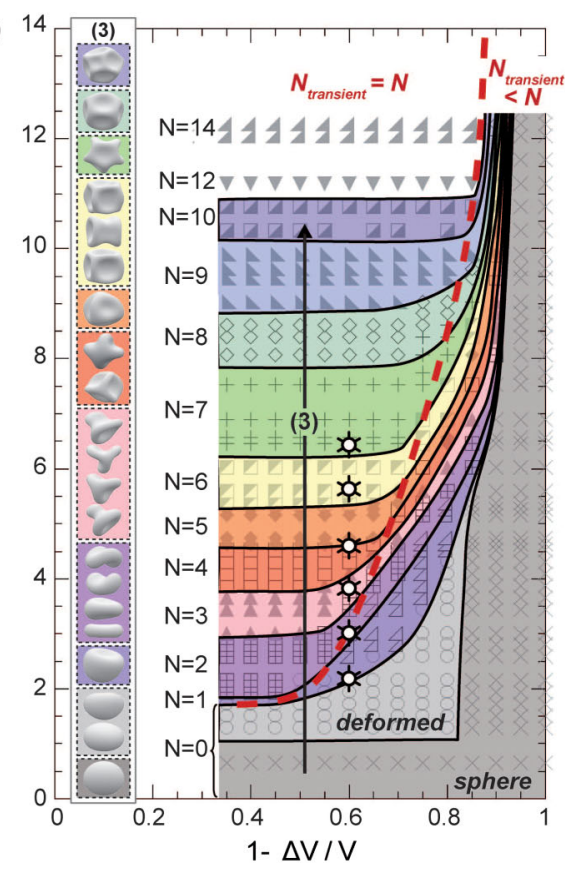

FIG. 4 (color online). Shape diagrams established from SURFACE EVOLVER simulations (gray points): number of depressions $N_{\text {transient }}$ or $N$ as a function of $v_{r}$, and $R / d_{\text {eq }}$ for 3 Poisson's ratios: $\nu_{2 \mathrm{D}}=0(\mathrm{a}), \nu_{2 \mathrm{D}}=0.5(\mathrm{~b}), \nu_{2 \mathrm{D}}=0.8$ (c). Dark and light gray zones correspond to conservation of the spherical symmetry and to deformations without appearance of depressions, respectively, and each colored zone to shapes with a given number of concave facets. The red dotted line delimits the zone where the number of depressions has reached its maximum value. In the transient zone, the shape may also evolve with $v_{r}$ as shown on insert (b): path (2) displays a sphere-discocyte-crisp evolution ( $v_{r}$ indicated under corresponding shapes); while path (1) shows cube becoming bulged cube on path (1). The universal sequence of Fig. 1, recalled and completed in insert (c), may be retrieved by following paths of type (3), within the $N_{\text {transient }}=N$ zone at any $\nu_{2 \mathrm{D}}$. experimental points obtained from the set of data at $v_{r}=0.6$; for this latter the vesicle radius is averaged for each $N$ and adimensionalized by the $d_{\text {eq }}$ obtained in Fig. 3.

giving quantitative clues on relative elastic features of gelphase lipid vesicles through mere observations, this study offers interesting insights into the structured, reproducible and stable shapes, that can be obtained through the deformation of simple soft objects.

We thank B. Audoly, K. Brakke G. Coupier, L. Mahadevan, G. Maret, P. Marmottant, and V. Vitkova for constructive interactions. F. Q. thanks IRTG "Soft Condensed Matter: Physics of Model Systems", DAAD, UFA-DFH Saarbrüucken and Universities of Konstanz, Strasbourg, Grenoble, and Aix-Marseille for funding.

*Catherine.Quilliet@ujf-grenoble.fr

†brigitte.pepin-donat@cea.fr

[1] F. Lautenschläger, S. Paschke, S. Schinkinger, A. Bruel, M. Beil, and J. Guck, Proc. Natl. Acad. Sci. U.S.A. 106, 15696 (2009).

[2] W. Helfrich, Z. Naturforsch. C 28, 693 (1973).

[3] H.-G. Dobereiner, E. Evans, M. Kraus, U. Seifert, and M. Wortis, Phys. Rev. E 55, 4458 (1997).

[4] R. Dimova, B. Pouligny, and C. Dietrich, Biophys. J. 79, 340 (2000).

[5] F.E. Antunes, E. F. Marques, M. G. Miguel, and B. Lindman, Adv. Colloid Interface Sci. 147-148, 18 (2009).
[6] R. L. Knorr, M. Staykova, R. S. Gracia, and R. Dimova, Soft Matter 6, 1990 (2010).

[7] B. T. Stokke, A. Mikkelsen, and A. Elgsaeter, Biophys. J. 49, 319 (1986).

[8] R. Mukhopadhyay, G. Lim, and M. Wortis, Biophys. J. 82, 1756 (2002).

[9] G. L. Lim, M. Wortis, and R. Mukhopadhyay, Soft Matter, Vol. 4: Lipid bilayers and red blood cells, edited by G. Gompper and M. Schick (Wiley-VCH Verlag GmbH \& Co. KGaA, Weinheim, Germany, 2008).

[10] E. Helfer, S. Harlepp, L. Bourdieu, J. Robert, F. C. MacKintosh, and D. Chatenay, Phys. Rev. Lett. 87, 088103 (2001).

[11] N. Delorme and A. Fery, Phys. Rev. E 74, 030901 (2006).

[12] M. I. Angelova, S. Soleau, P. Meleard, J.-F. Faucon, and P. Bothorel, Prog. Colloid Polym. Sci. 89, 127 (1992).

[13] R. Koynova and M. Carey, Biochim. Biophys. Acta 1376, 91 (1998).

[14] D. Needham and E. Evans, Biochemistry 27, 8261 (1988).

[15] H.-G. Dobereiner, Current Opinion in Colloid \& Interface Science 5, 256 (2000), and references therein.

[16] L. D. Landau and E. M. Lifshitz, Course of Statistical Physics, Vol. 7: Theory of Elasticity (ButterworthHeinemann, Oxford, 1986), 3rd ed.

[17] J. Lidmar, L. Mirny, and D. R. Nelson, Phys. Rev. E 68, 051910 (2003). 
[18] G. A. Vliegenthart and G. Gompper, New J. Phys. 13, 045020 (2011).

[19] C. Quilliet, C. Zoldesi, C. Riera, A. van Blaaderen, and A. Imhof, Eur. Phys. J. E 27, 13 (2008), and erratum.

[20] T. T. Nguyen, R. F. Bruinsma, and W. M. Gelbart, Phys. Rev. E 72, 051923 (2005).

[21] See Supplemental Material at http://link.aps.org/ supplemental/10.1103/PhysRevLett.108.108303 for energy considerations.

[22] E. Katifori, S. Alben, E. Cerda, D. R. Nelson, and J. Dumais, Proc. Natl. Acad. Sci. U.S.A. 107, 7635 (2010).
[23] T. Kaasgaard, C. Leidy, J. H. Crowe, O. G. Mouritsen, and K. Jorgensen, Biophys. J. 85, 350 (2003).

[24] We consider that mutual sliding of the monolayers at the micron scale is prevented by friction at the ripples edges. Then, contrary to fluid bilayers that may require the ADE model, we model out-of plane deformations of the gel bilayer by a single surface with a Helfrich curvature energy. The spontaneous curvature $C_{0}$ slightly changes from $15^{\circ} \mathrm{C}$ to $23.6{ }^{\circ} \mathrm{C}$ but in no case $C_{0}$ will exceed the Lobkovski limit $L^{-1} \gamma(L)^{1 / 6}\left(L=R / \sqrt{N} \approx \sqrt{R d_{\mathrm{eq}}}\right.$ is the length of the rims between concave facets), above which $C_{0}$ could have a significant impact on the vesicle shape [20]. 